\title{
Infant and Young Child Feeding Practices and Associated Factors Among Children Aged 0-23 Months in Assayita District Afar Region Ethiopia
}

\author{
Molla Kahssay ${ }^{1, ~ *, ~ E d r i s ~ E b r a h i m ~}{ }^{2}$, Oumer Seid ${ }^{3}$, Etsay Woldu ${ }^{1}$, Surender Reddy $^{1}$ \\ ${ }^{1}$ Department of Public Health, Samara University, Afar, Ethiopia \\ ${ }^{2}$ Unicef Branch Offices, Samara, Afar, Ethiopia \\ ${ }^{3}$ School of Public Health, Bahirdar University, Bahirdar, Amhara, Ethiopia
}

Email address:

Mollaka2005@gmail.com (M. Kahssay), edrisebrahim132@gmail.com (E. Ebrahim), oumer0918@gmail.com (O. Seid), etsaywold@gmail.com (E.Woldu),dr.surenderreddy@yahoo.com (R. Surender)

${ }^{*}$ Corresponding author

\section{To cite this article:}

Molla Kahssay, Edris Ebrahim, Oumer Seid, Etsay Woldu, Surender Reddy. Infant and Young Child Feeding Practices and Associated Factors Among Children Aged 0-23 Months in Assayita District Afar Region Ethiopia. Journal of Food and Nutrition Sciences. Vol. 7, No. 6, 2019, pp. 96-104. doi: 10.11648/j.jfns.20190706.13

Received: August 9, 2019; Accepted: October 24, 2019; Published: November 26, 2019

\begin{abstract}
Achieving optimum Infant and young child feeding practices is the major challenge in developed and developing countries. Globally, about $40 \%$ of under two years age deaths are attributed to inappropriate infant and young child feeding practices. In Ethiopia, a wide range of inappropriate infant and young child feeding practices were documented. The study was aimed to assess infant and young child feeding practice and its associated factors among children aged 0-23 months in Assayita districts, Afar region, Ethiopia, 2018. A Community based cross-sectional study was applied from January1-30/2018 among 620 study participants. A pre tested structured questioner was used to collect data. After data get collected it was cleaned and entered using EPI-Data version-3.02 and exported to SPSS version-20 for further analysis. Binary logistic regression analysis was used to measure the strength of association between explanatory variables and outcome variable. Variables with $\mathrm{p}<0.25$ on univariable logistic regression analysis were candidates for multivariable logistic regression analysis and statistical significance was declared at P-value $<0.05$ and $95 \%$ CI. In this study the prevalence of appropriate infant and young child feeding practice was $9.2 \%$ (95\% CI. 7.1-11.6), children from mothers with secondary education (AOR=4.44, 95\% CI (1.84, 10.7), delivered at health facilities $(\mathrm{AOR}=2.55,95 \%$ CI $(1.32,4.93)$, had Ante Natal Care follow-up (AOR=4.2, 95\% CI (2.2, 8.7), and heard information about Infant and young child feeding $(\mathrm{AOR}=4.38,95 \%$ CI $(1.97,9.5)$ were predictors of appropriate Infant and young child feeding practice at 95\% CI. Promoting institutional delivery, promoting Ante Natal Care service, maternal education and increasing awareness on infant and young child feeding practice should be implemented.
\end{abstract}

Keywords: Infant Young Child Feeding Practice, Assayita, Afar, Samara University

\section{Introduction}

Infant and young child feeding (IYCF) in the first two years of life is base for growth and development of children. [1] The first 1000 days of life from conception through the first two years of life is a window of opportunity for child development with optimal nutrition and it also avoids damage because the during this period is often permanent. [2] The global strategy for infant and young child feeding describes essential actions to protect, promote and support appropriate infant and young child feeding. It focuses on the importance of investing in this crucial area to ensure that children grow to their full potential free from the adverse consequences of compromised nutritional status and preventable illnesses. [3] Under nutrition usually occurs at the age of 3-18 months, hence the child's first two years of life are considered a critical window of opportunity for the prevention of growth retardation and under nutrition, therefore age appropriate interventions should be taken to 
ensure that children reach their full growth potential and to prevent irreversible stunting and acute under nutrition. [4, 5]

Promotion of exclusive breastfeeding is the single most cost-effective intervention to reduce infant morbidity and mortality in developing countries. Even after complementary foods have been introduced at $6^{\text {th }}$ month, breastfeeding remains a critical source of nutrients for the infant and young child. It provides about one half of an infant's energy needs up to the age of one year, and up to one third during the second year of life. Breast milk continues to supply higher quality nutrients and protective factors then complementary foods. Therefore, breastfeeding recommended up to 2 years and beyond with adequate complementary feeding. [6-8]

Achieving optimum IYCF practices is the major challenge in developing and developed countries [9]. To improve, maintain and promote IYCF practices, the World Health Organization (WHO) recommended eight core indicators. Early initiation of breastfeeding, Exclusive breastfeeding under 6 months, Continued breastfeeding at 1 year, Introduction of solid, semi-solid or soft foods, Minimum dietary diversity, Minimum meal frequency, Minimum acceptable diet and Consumption of iron-rich or iron-fortified foods are those eight indicators. WHO also recommended seven optional indicators such as Children ever breastfed, Continued breastfeeding at 2 years, Age-appropriate breastfeeding, Predominant breastfeeding under 6 months, Duration of breastfeeding, Bottle feeding and Milk feeding frequency for non-breastfed children. [10]

Globally, about $40 \%$ of under two years deaths are associated with inappropriate feeding practices. [10] Optimal breastfeeding and appropriate complementary feeding could prevent $13 \%$ and $6 \%$ under-five mortality respectively. About two third of malnutrition associated with inappropriate feeding practices during the first year of life. [11] Every day, 3000 - 4000 infants die in the developing world from diarrhea and acute respiratory infections because they are given inadequate amounts of breast milk. More than 10 million children die each year in sub Saharan Africa and South Asia. A major contributor to their deaths is poor breastfeeding practices. The risk of death from diarrhea of partially breastfed infants 0 - 6 months of age was 8.6 times more risk than exclusively breastfed children. [12]

In low- and middle-income countries, recent data revealed that optimal breast feeding prevents about $12 \%$ of under- 5 child mortality every year. [13] Other contemporary studies in Ethiopia, Ghana, Bolivia and Madagascar have shown that breast feeding prevents $20-22 \%$ of neonatal deaths. [14-16] Suboptimal IYCF practices increase the risk of infant and child morbidity and mortality by up to five fold. [17] Child malnutrition causes approximately 2.7 million deaths per year, 156million stunting, 50million wasting and 42million overweight or obesity. [18] In developing countries, approximately $25-50 \%$ of infant mortality is attributed to suboptimal IYCF practices. [19-21] The Central Statistical Agency (CSA) 2016 report indicated that the neonatal mortality rate accounts for $43 \%$ of under- 5 mortality in Ethiopia. [22]

A wide range of harmful infant and young child feeding practices were documented in Ethiopia. According to Ethiopian Demographic and Health Survey of 2016, $73 \%$ of infants started breastfeeding within one hour of birth and $58 \%$ infants practiced exclusive breastfeeding during the first six months. About half (60\%) of children aged $6-8$ months consumed solid, semi-solid, or soft foods and $14 \%$ of children were fed minimum dietary diversity and $45 \%$ of children fed minimum meal frequency per day while $92 \%$ of children continued breastfeeding at one year, and $76 \%$ continued at 2 year. Only $67 \%$ children under the age of two received age-appropriate breastfeeding. The pre-lacteal feeds within the first three days of life were $8 \%$, while $14 \%$ used a bottle with a nipple. [22]

To optimize National IYCF practices, the Ethiopian government has been training health professionals, developing and revising procedural manuals and implementing the community integrated management of childhood illnesses and Baby-friendly Hospital Initiative program based on expert opinion, International research and adapted tools accordingly. [23-25] several systematic reviews and meta-analyses have been conducted on the time of initiating of breast feeding (TIBF) [26], exclusive breast feeding [9], long-term effects of breast feeding [27], breast feeding and intelligence [28], duration of exclusive breast feeding [29] and effectiveness of complementary feeding [30]. However, these efforts have failed to bring about substantive and sustainable changes leading to improvement of infant and young child feeding practices. There are limited studies regarding infant and young child feeding practice and associated factors with inappropriate feeding practices in the study area. Therefore, this study was aimed to assess infant and young child feeding practice and associated factors among mothers of children aged 0-23 months in Assayita district, Afar region, Ethiopia.

\section{Methods}

\subsection{Study Area and Period}

The study was conducted in Assayita district which is one of 32 districts in Afar. It is $655 \mathrm{kms}$ far away from the capital city Addis Ababa and $70 \mathrm{kms}$ far from the capital city of Afar, Samara. According to the regional administrative report, total population of the districts is 58,274 and the total population of under 5 children is 7645 with a total of 11,655 households. Livestock production is the dominant livelihood source. As of the report from the ARHB, the district has one hospital, one health center, 10 health posts, and 4 private clinics with a total no of 20 health extension workers. The study was conducted from January1-30/2018.

\subsection{Study Design}

Community based cross-sectional study design.

\subsection{Eligibility Criteria}

\subsubsection{Inclusion Criteria}

All mothers having children aged 0-23 months of age in 
the selected kebelles were part of this study.

\subsubsection{Exclusion Criteria}

Mothers who were seriously ill and mothers who were unable to communicate were excluded from this study

\subsection{Sample Size Determination}

The sample size was determined by using single population proportion formula

$$
\mathrm{n}=\frac{(\mathrm{Z} \alpha / 2)^{2} \times \mathrm{P}(1-\mathrm{P})}{d^{2}}
$$

Considering the following assumptions:

A Prevalence of exclusive breastfeeding practice 52\% [31].

$\mathrm{Z} \alpha / 2=$ critical value at $95 \%$ Confidence level of certainty (1.96).

$\mathrm{d}=$ marginal error $5 \%$.

$$
\mathrm{n}=\frac{1.96^{2} \times 0.52 \times 0.48}{0.05^{2}}=383
$$

Using 1.5 design effect and 10\% non-response rate the final sample size was 632 .

\subsection{Sampling Procedure}

Districts were stratified to urban and rural kebelles and four rural and 2 urban kebelles were selected randomly. Next to this, number of households was proportionally allocated to each selected kebelles according to their population proportion to size. Finally systematic random sampling technique was used to get the required samples from each selected kebelle.

\subsection{Operational Definition}

Optimal infant and young child feeding: initiation of breastfeeding within one hour of birth, exclusively breastfed for the first six months, after which nutritiously appropriate, adequate, and safe complementary foods should be introduced along with continuing breastfeeding up to two years and beyond [8].

Appropriate infant and young child feeding practice: Exclusive breast feeding in children age less than 6 months, early initiation of breast feeding (within one hour), minimum meal frequency ( $>=2$ for children aged 6 to 8 months, $>=3$ for children aged 9 to 23 months in breast feeding children) and ( $>=4$ for children aged 6 to 23 months in non-breast feeding children), minimum dietary diversity $(>=4)$, timely introduction of solid, semi-solid and soft foods in 6 - 8 months and breast feeding, minimum acceptable diet, consumption of iron-rich or iron-fortified foods and continued breast feeding up to one year (12-15 months). A practice is considered as appropriate if a specific age group received a score of 1 , and a practice that is considered as inappropriate if received a score of 0 . If summed score of the indicators is equal to 4 or above, it was considered as appropriate IYCFP and if it is below 4 it is inappropriate [36].
Complementary foods: any solid or semi-solid or soft foods which are given to the child. Timely initiation of breast feeding: Putting neonate on mother breast to suck within 1 hour of delivery (including 1hour) [7, 8].

Ever breast feeding: breast feeding any time since birth

Minimum dietary diversity: children 6 - 23 months of age who consume 4 or more food groups from 7 food groups with 24 hours dietary recall.

Continued breast feeding at 1 year: children 12 - 15 months of age who had breast feed $[3,7]$.

Continued breast feeding at 2 year:-children $20-23$ months of age who had breast milk fed.

Exclusive breast-feeding (EBF): Infants, who received only breast-milk from his/her mother, and no other liquids or solids with the exception of drops or syrup consisting of vitamins, mineral or medicines. EBF measured for child less than 6 months if the child only breast fed within 24 hour and if child is greater than 6 months $[7,8]$.

Minimum acceptable diet: -a composite indicator of minimum dietary diversity and minimum meal frequency. It is considered if the currently breastfed and non-breast fed child meets both the minimum diversity and the minimum meal frequency $[7,8]$.

Minimum meal frequency: - children age 6 - 23 months who receive solid, semi-solid, or soft foods the minimum number ( 3 times for breastfed children and 4 times for nonbreastfed children with 24 hours dietary recall [7, 8].

Predominant breast feeding: the infants' predominant nourishment with breast-milk and allows the infant to receive certain liquids (water and water-based drinks, fruit juice), ritual fluids and ORS, drops or syrups (vitamins, minerals, medicines). Non-human milk and food-based fluids are not allowed $[7,8]$.

Pre lacteal feeding: -children have given something other than breast milk during the first three days of life.

\subsection{Data Collection Tools}

Pretested structured questionnaire was used to collect the data. The data was collected by four diploma nurses who are proficient in writing and speaking both Amharic and the local language Afargna with close supervision by supervisor and the researcher.

\subsection{Data Collection Procedure and Quality Control}

The questioner was prepared in English and translated in to Amharic and local language Afaraff. Data collectors and supervisors were trained for two days on the objective of study, content of questionnaire, how to help study participants and how to take consent from respondents in order to ensure quality of data. The developed questionnaires was pretested on $5 \%$ of sample size and based on feedback from pre-test, necessary modifications were done.

\subsection{Data Management, Analysis and Interpretation}

At the end of data collection, data was checked for completeness, entered in to Epi- data software for cleaning 
and transported to SPSS for analysis. Descriptive analysis was used to see frequency, and percentages of the characteristics. Binary logistic regression was used to assess significant predictors of the outcome variable. Variables having $\mathrm{P}$-value $=<0.25$ in univariable binary logistic regression were taken to multivariable logistic regression to control confounding effect. Finally, Odd Ratio (OR) and 95\% confidence intervals (CI) were used to express the final mode and statistical significance was declared at $\mathrm{P}$-value $=<0.05$.

\subsection{Ethical Consideration}

Ethical clearance was obtained from Samara University Ethical Review Committee and Permission to conduct the study was obtained from Afar regional health bureau, Assayita districts health office. Oral informed consent was obtained from mothers after describing the benefits of the study and participant's involvement in the study was on voluntary basis and participants who would be unwilling to participate in the study and those who wish to quit their participation at any stage were informed to do so without any restriction.

\section{Results}

\subsection{Socio Demographic Characteristics of Respondents}

A total of 620 study participants were included in the study with response rate of $98.1 \%$. Three hundred forty five $(55.6 \%)$ of the study subjects were female and $259(41.8 \%)$ of them were between 12-23 months old with the mean age of $10.1( \pm 6.39 \mathrm{SD})$ months. Two hundred seventy nine $(45 \%)$ of the study subject were with birth interval $<24$ months. The ages of the study participants were between 20-34 (76\%) years with the mean age of $26.35( \pm 5.54 \mathrm{SD})$ years and 349 $(56.3 \%)$ of them were with no education. Five hundred fifty two $(89 \%)$ mothers of the study subjects were married. With regard to religion, $561(90.5 \%)$ of the study participant were Muslim and 489 (78.9\%) of the households have monthly income $>1000$ birr per month (Table 1).

Table 1. Socio demographic characteristics of study subjects in Assayita district, Afar region, Ethiopia, $2018(n=620)$.

\begin{tabular}{lll}
\hline Variables & $\mathbf{N}$ & $\mathbf{\%}$ \\
\hline Age of child in months & & \\
$<6$ & 196 & 31.6 \\
$6-11$ & 165 & 26.6 \\
$12-23$ & 259 & 41.8 \\
Sex & & \\
Male & 275 & 44.4 \\
Female & 345 & 55.6 \\
Birth order & & \\
$1^{\text {st }}$ & 157 & 25.4 \\
$2-4$ & 389 & 62.7 \\
$>4$ & 74 & 11.9 \\
Birth interval & & \\
No previous birth & 157 & 25.3 \\
$<24$ & 279 & 45 \\
$>=24$ & 184 & 29.7 \\
Maternal age & & \\
$15-19$ & 109 & 17.5 \\
\hline
\end{tabular}

\begin{tabular}{lll}
\hline Variables & $\mathbf{N}$ & $\mathbf{\%}$ \\
\hline $20-34$ & 471 & 76 \\
$>34$ & 40 & 6.5 \\
Marital status & & \\
Married & 552 & 89 \\
Divorced & 35 & 5.6 \\
Single & 33 & 5.4 \\
Religion & & \\
Muslim & 561 & 90.5 \\
Orthodox & 59 & 9.5 \\
$<=1000$ & 131 & 21.1 \\
$>1000$ & 489 & 78.9 \\
\hline
\end{tabular}

\subsection{Reproductive Health Services and Child Health Characteristics of Respondents}

Three hundred sixty four $(58.7 \%)$ of women were delivered their current child at home and 256 (41.3\%) of them were had ANC follow up. With regard to postnatal care services, $218(35.2 \%)$ of mothers of the study subjects got the services. Three hundred two $(48.7 \%)$ of mothers of the study subject had 3-5 number of pregnancies. with regard to information related to IYCF service, $388(62.6 \%)$ of mother had heard the information from different sources (Table 2).

Table 2. Reproductive health services and child health characteristics among study participants in Assayita district, Afar region, Ethiopia, 2018 $(n=620)$.

\begin{tabular}{lll}
\hline Variables & N & $\%$ \\
\hline Place of delivery & & \\
Home & 364 & 58.7 \\
Health facilities & 256 & 41.3 \\
Mode of delivery & & \\
Normal & 475 & 76.6 \\
C/s & 145 & 23.4 \\
ANC follow up & & \\
Yes & 256 & 41.3 \\
No & 364 & 58.7 \\
Number of ANC visit & & \\
$1^{\text {st }}$ & 72 & 28.1 \\
$2-3$ & 112 & 43.8 \\
>=4 & 72 & 28.1 \\
Counselled on IYCFP & & \\
Yes & 179 & 69.9 \\
No & 77 & 30.1 \\
PNC & & \\
Yes & 218 & 35.2 \\
No & 402 & 64.8 \\
Counselled on breast feeding & & \\
Yes & 195 & 89.4 \\
No & 23 & 10.6 \\
Child sick in the last 2 wks. & & \\
Yes & 131 & 37.4 \\
No & 489 & \\
Heard about IYCF information & 388 & \\
Yes & 232 & \\
No & & \\
\hline & & \\
\hline
\end{tabular}

\subsection{Child Feeding Practice Characteristics}

Six hundred twenty $(100 \%)$ of the study subject were ever breast fed and $343(55.5 \%)$ of study subjects initiated breast feeding within one hour interval. Five hundred ninety one $(95.3 \%)$ of the study subjects fed colostrum. Six hundred two $(97.1 \%)$ of the study subject were currently breast feeding 
and $315(52.3 \%)$ of study subject breast fed $>=8$ times per day. One hundred thirty four $(68.4 \%)$ of the children were exclusively breast fed from $0-5$ months old and the mean age of exclusive breast feeding was $4.35( \pm 1.48 \mathrm{SD})$ months and $119(19.2 \%)$ of the study subject were experienced prelacteal feeding (Table 3).

Table 3. Child feeding practice among children aged 0-23 months in Assayita district, Afar region, Ethiopia, 2018 ( $n=620)$.

\begin{tabular}{lll}
\hline Variables & N & \% \\
\hline child Ever breast feed & & \\
Yes & 620 & 100 \\
Current breast feeding & & \\
Yes & 602 & 97.1 \\
No & 18 & 2.9 \\
Pre lacteal feeding & & \\
Yes & 119 & 19.2 \\
No & 501 & 80.8 \\
Bottle feeding & & \\
Yes & 186 & 30 \\
No & 434 & 70 \\
Perceived importance of breast feeding & & \\
Growth and development & & \\
Yes & 485 & 78.2 \\
No & 135 & 21.8 \\
Prevent from disease & & \\
Yes & 399 & 64.4 \\
No & 221 & 35.6 \\
Immunity & & 63.5 \\
Yes & 394 & 36.5 \\
No & 226 & \\
\hline & & \\
\hline
\end{tabular}

\subsection{Child Complementary Feeding Practice of Respondents}

Three hundred twenty nine $(68.7 \%)$ of the study subjects started semisolid foods at $>=6$ months and 98 (100\%) of breast feeding children ( 6 to 8 months) had minimum meal frequency $>=2$ times. Sixty eight $(69.4 \%)$ of the study subject were introduced solid, semisolid and soft food between 6 to 8 months. Fifty seven $(9.2 \%)$ of the study subjects had appropriate IYCF practices (Table 4).

Table 4. Child complementary feeding practices among children aged 0-23 months in Assayita district, Afar region, Ethiopia, $2018(n=620)$.

\begin{tabular}{lcc}
\hline Variables & $\mathbf{N}$ & $\%$ \\
\hline \multicolumn{2}{l}{ Age of initiating semisolid food } & \\
$<6$ months & 150 & 31.3 \\
$>=6$ months & 329 & 68.7 \\
Thickness of food & & \\
Thin like soap & 214 & 45.1 \\
medium thickness & 235 & 49.5 \\
Very thick & 26 & 5.5 \\
Minimum meal frequency $(9-23$ months breast feed per $24 \mathrm{hr})$ \\
$<3$ & 135 & 43.8 \\
$>=3$ & 173 & 56.2 \\
Minimum meal frequency $(6-8$ months breastfeed per 24 hrs $)$ \\
$<2$ & 0 & 100 \\
$>=2$ & 98 & \\
Minimum meal frequency $(6-23$ months none breastfeed $)$ & 66.7 \\
$<4$ & 12 & 33.3 \\
$>=4$ & 6 & 100 \\
Minimum acceptable diet & 424 & \\
No & 0 & \\
Yes & & \\
\hline
\end{tabular}

\begin{tabular}{|c|c|c|}
\hline Variables & $\mathbf{N}$ & $\%$ \\
\hline \multicolumn{3}{|c|}{ Introduction solid, semisolid and soft food (6-8 months breast feed) } \\
\hline No & 30 & 30.6 \\
\hline Yes & 68 & 69.4 \\
\hline \multicolumn{3}{|c|}{ Child feed Flesh foods (meat, fish, liver/organ meats) per $24 \mathrm{hr}$} \\
\hline No & 229 & 56.4 \\
\hline Yes & 177 & 43.6 \\
\hline \multicolumn{3}{|c|}{ Continued breast feeding up to 1 year (12-15 moths) } \\
\hline No & 7 & 6.3 \\
\hline Yes & 105 & 93.7 \\
\hline \multicolumn{3}{|l|}{ IYCF practice } \\
\hline Appropriate & 57 & 9.2 \\
\hline In appropriate & 563 & 90.8 \\
\hline
\end{tabular}

\subsection{Minimum Dietary Diversity Practice}

Fifty four $(13.3 \%)$ of the children had minimum dietary diversity score $>=4$ within 24 hours prior to the survey date and $230(56.7 \%)$ of the study subjects have consumed grains, roots and tubers food groups and $375(92.4 \%)$ dairy products within 24 hours prior to the study period.

Table 5. Minimum dietary diversity among 6-23 months old breast feeding children in Assayita district, Afar region, Ethiopia, $2018(n=620)$.

\begin{tabular}{|c|c|c|}
\hline Variables & $\mathbf{N}$ & $\%$ \\
\hline \multicolumn{3}{|c|}{ Grain, roots and tubers } \\
\hline No & 176 & 43.3 \\
\hline Yes & 230 & 56.7 \\
\hline \multicolumn{3}{|c|}{ Dairy product } \\
\hline No & 32 & 7.6 \\
\hline Yes & 375 & 92.4 \\
\hline \multicolumn{3}{|c|}{ legumes and nuts } \\
\hline No & 92 & 22.7 \\
\hline Yes & 314 & 77.3 \\
\hline \multicolumn{3}{|l|}{ Eggs } \\
\hline No & 327 & 80.5 \\
\hline Yes & 79 & 19.5 \\
\hline \multicolumn{3}{|l|}{ Flesh foods } \\
\hline No & 229 & 56.4 \\
\hline Yes & 177 & 43.6 \\
\hline \multicolumn{3}{|c|}{ Vitamin A rich fruits } \\
\hline No & 379 & 93.3 \\
\hline Yes & 27 & 6.7 \\
\hline \multicolumn{3}{|c|}{ Others fruits and vegetables } \\
\hline No & 386 & 95.1 \\
\hline Yes & 20 & 4.9 \\
\hline \multicolumn{3}{|c|}{ Minimum dietary diversity (6-23 months per $24 \mathrm{hrs}$ ) } \\
\hline$<4$ & 352 & 86.7 \\
\hline$>=4$ & 54 & 13.3 \\
\hline
\end{tabular}

\subsection{Factors Associated with Infant and Young Child Feeding Practices}

The selected variables were tested their individual contribution for infant and young child feeding through univariable logistic regression analysis. Then variables with $\mathrm{p}-$ value $<0.25$ were entered together to determine their effect on the outcome variable IYCF practice on multivariable logistic regression analysis and found that being educated mothers with secondary education ( $\mathrm{AOR}=4.44,95 \% \mathrm{CI}(1.84,10.7)$, delivered at facilities (AOR $=2.55,95 \% \mathrm{CI}(1.32,4.93))$, had ANC follow-up $(\mathrm{AOR}=4.2,95 \% \mathrm{CI}(2.2,8.7))$, and heard information about IYCF $(\mathrm{AOR}=4.38,95 \% \mathrm{CI}(1.97,9.5))$ were predictors of appropriate IYCF at $95 \% \mathrm{CI}$ (Table 6). 
Table 6. Logistic regression analysis output of factors affecting infant and young child feeding practice in Assayita district, Afar region, Ethiopia, 2018 $(n=620)$.

\begin{tabular}{|c|c|c|c|c|}
\hline \multirow{2}{*}{ Variables } & \multicolumn{2}{|l|}{ IYCF practice } & \multirow{2}{*}{ COR $(95 \%$ CI) } & \multirow{2}{*}{ AOR (95\% CI) } \\
\hline & Appropriate n (\%) & In appropriate n (\%) & & \\
\hline \multicolumn{5}{|l|}{ Maternal education } \\
\hline No formal education & $20(35.1)$ & $329(58.4)$ & 1 & 1 \\
\hline Primary & $10(17.5)$ & $66(11.7)$ & $2.49(1.12,5.5)$ & $1.79(0.66,4.8)$ \\
\hline Secondary & $17(29.8)$ & $110(19.5)$ & $2.54(1.28,5.1)$ & $4.44(1.84,10.7)^{*}$ \\
\hline Higher & $10(17.5)$ & $58(10.3)$ & $2.84(1.26,6.4)$ & $2.1(0.71,6.1)$ \\
\hline \multicolumn{5}{|l|}{ Husband education } \\
\hline No formal education & $15(30.6)$ & $174(34.6)$ & 1 & 1 \\
\hline Primary & $13(26.5)$ & $73(14.5)$ & $2.21(0.91,4.5)$ & $1.87(0.74,4.65)$ \\
\hline Secondary & $12(24.5)$ & $70(13.9)$ & $1.98(0.88,4.46)$ & $1.42(0.52,3.8)$ \\
\hline Higher & $9(18.4)$ & $186(37)$ & $0.56(0.24,1.32)$ & $0.32(0.16,1.01)$ \\
\hline \multicolumn{5}{|l|}{ Place of delivery } \\
\hline Home & $21(36.8)$ & $343(60.9)$ & 1 & 1 \\
\hline Health facility & $36(63.2)$ & $220(39.1)$ & $2.67(1.52,4.69)$ & $2.55(1.32,4.93)^{*}$ \\
\hline \multicolumn{5}{|l|}{ ANC } \\
\hline Yes & $40(70.2)$ & $216(38.4)$ & $3.78(2.1,6.8)$ & $4.2(2.2,8.7)^{*}$ \\
\hline No & $17(29.8)$ & 347 (61.6) & 1 & 1 \\
\hline \multicolumn{5}{|l|}{ PNC } \\
\hline Yes & $27(47.4)$ & $191(33.9)$ & $1.75(1.01,3.03)$ & $0.83(0.41,1.67)$ \\
\hline No & $30(52.6)$ & $372(66.1)$ & 1 & 1 \\
\hline \multicolumn{5}{|l|}{ Heard about IYCF } \\
\hline Yes & $43(75.4)$ & $345(61.3)$ & $1.94(1.04,3.63)$ & $4.38(1.97,9.5)^{*}$ \\
\hline No & $14(24.6)$ & $218(38.7)$ & 1 & 1 \\
\hline
\end{tabular}

$\mathrm{COR}=$ Crude odds ratio, $\mathrm{AOR}=\mathrm{Adjusted}$ odds ratio, $\mathrm{CI}=$ confidence interval, *significant at $\mathrm{p}<0.05$.

\section{Discussion}

This study assessed infant and young child feeding practice and associated factors among children aged 0-23 months in Assayita district Afar region Northeast Ethiopia and the magnitude of appropriate infant and young child feeding practice was found to be $9.2 \%$.

The WHO guidelines on infant and young child feeding practices do not provide the baseline or neither minimum standard that needs to be reached nor what percentage should be considered alarming for public health significance. [7-8] logically, it is desirable that all children $0-23$ months meet the recommended feeding practices. To compare the similarities and differences of the prevalence and factors associated of infant and young child feeding practice with other studies are difficult due to lack of study on IYCF practices in composite of similar indicators. These study findings were discussed based on the individual components of IYCF. Despite this limitation, we tried to discuss the prevalence and associated factors with infant and young child feeding practice by considering appropriate IYCFP (if study subject use $50 \%$ or above $(>=4)$ of the 8 core WHO indicators IYCF).

The prevalence of appropriate IYCF practice in this study was lower than study conducted in Shashemene which is $32.1 \%$. [32] The difference might be due to socioeconomic and cultural difference between the study subjects. This study showed the prevalence of ever breast fed was $100 \%$. This was nearly similar to study done in Mekelle town $98.9 \%$ [33], Shshemene 99.3\%, Arbaminch which was 95.8\%. [34] This high rate of breast feeding in study area may be due to the fact that breast feeding practice is common and normal among the society.

Despite the fact that WHO Global and National Infant, and Young Child Feeding Guidelines recommend that all newborns should start breastfeeding immediately within the first hour after delivery [7], our study finding revealed that $55.3 \%$ of study subjects initiate breast feeding within one hour interval after birth. This was similar with study done in Shashemene 58.0\% [32] and EDHS 2011, 52\% [22]. However, this finding was lower than study done in Mekelle $78 \%$, Bahirdar 85\% [35] and Asela 86.3\%. [36] The difference might be due to socio-cultural difference or lack of knowledge about the initiation of breast feeding.

This study finding revealed that the prevalence of exclusive breastfeeding for infants less than six months from 0-5 months old was $68.4 \%$ and the mean age of exclusive breast feeding was $4.35( \pm 1.47 \mathrm{SD})$ months which is contrary to recommendation by WHO that all children under age 6 months should be exclusively breastfed. [7] This is higher than EDHS 2016, 58\% [38], Mekelle town 60.8\%, However, the study finding was lower than study done in Shashemene $87.8 \%$ and Asela 77\%. Our study finding showed the prevalence of bottle feeding was $30 \%$. This was higher than study done in shashemene $20.9 \%$, Bahirdar 23\% and Western Uganda $10 \%$ [37].

Timely introduction of nutritionally adequate and safe complementary foods promotes growth and good nutritional status among infants and young children. Our study finding revealed that, $69.4 \%$ of children $6-8$ months received solid, semisolid and soft foods. This was similar to study done in shashemene $65.7 \%$. However this finding was lower than study done in Uganda (75\%), Tanzania (92.3\%) [38] and 
Kenya (81\%). [11] This may be due to the difference in mothers' knowledge on what and when to start additional foods to child and their perception that the child is unable to digest foods in this age in the study areas.

This study showed that $98(100 \%)$ children aged 6- 8 months received requirements for minimum meal frequency $(>=2)$ and children 9-23 months received 56.2\% requirements for minimum meal frequency $(>=3)$. However, $33.3 \%$ of non-breast fed children aged $6-23$ months received minimum dietary diversity within 24 hour prior to the survey $(>=4)$. This is lower than study done in Tanzania $38 \%$ of children age received minimum dietary diversity. Our study finding was also higher than study done in South Asia; the minimum meal frequency was less than $50 \%$ in most of south Asia countries. [39]

Our study findings revealed that consumption of flesh foods like meat and iron rich foods was $43.6 \%$. This finding is higher than study finding in shashemene. This difference might be due to difference in the study settings.

With regard to factors associated with infant and young child feeding the multivariable logistic regression analysis revealed that mothers with secondary education $(\mathrm{AOR}=4.44$, $95 \% \mathrm{CI}(1.84,10.7)$, delivered at facilities $(\mathrm{AOR}=2.55,95 \%$ CI $(1.32,4.93)$, had ANC follow-up $(\mathrm{AOR}=4.2,95 \% \mathrm{CI}(2.2$, 8.7), and heard information about IYCF $(\mathrm{AOR}=4.38,95 \% \mathrm{CI}$ $(1.97,9.5)$ were predictors of appropriate IYCF at $95 \%$ CI.

This study finding revealed that, infant and young children's from educated mothers with secondary level education were 4.4 time more likely to have appropriate IYCF practice than infant and young children's from noneducated mothers $(\mathrm{AOR}=4.44,95 \%$ CI $(1.84,10.7)$. This might be due to the fact that, educated mother would have better skills and information for planning purposes as well as for implementing strategies that can meet adequately the nutritional needs of children and the whole family. Moreover, mothers with better education are more likely to have acquired a better childcare skill that is essential to meet the nutritional needs of their children. [40]

Our study also showed that study subject whose mothers had ANC follow-up were 4.2 times more likely to have appropriate feeding than their counterpart $(\mathrm{AOR}=4.2,95 \%$ CI $(2.2,8.7)$. This is similar to study done in shashemene. This might be because of lack of antenatal visit might create favorable environment for traditional child feeding malpractices that might affect child nutritional status. In contrast to this, mothers who had attended antenatal visit might have advised by health professionals on proper child feeding practices.

This study revealed that, infant and young children's delivered at facility level were more likely to have appropriate IYCF practice than their counterpart (AOR=2.55, $95 \%$ CI $(1.32,4.93)$. This is in line with study done in shashemene. This could be explained by the fact that mothers who delivered at health facility could have got breast and complementary feeding advice.

Our finding also revealed that study participant who had heard about IYCF information were 3.88 times more likely to have appropriate IYCF Practices than their counterpart $(\mathrm{AOR}=4.38,95 \% \mathrm{CI}(1.97,9.5)$. This is in line with study on analysis of $2005 \& 20111$ Ethiopia demographic and health surveys. [41]

\subsection{Strength of the Study}

We have used primary data Source.

\subsection{Limitation of the Study}

Due to the fact that the study was cross sectional study describing cause and effect relationship of the exposure and outcome variables was difficult.

\section{Conclusion}

The overall prevalence of appropriate infant and young child feeding practice was $9.2 \%$. Infant and young child feeding practice in the community is still very poor. Children from a mother with secondary education, children from mothers delivered at facilities, children from mothers had ANC follow-up and heard information about IYCF were important predictors of appropriate infant and young child feeding practices.

\section{Recommendation}

The regional health bureau and Asita district health office should increase awareness creation on mothers particularly using the health extension workers and community social mobilization on components or indicators of infant and young child feeding.

Strengthen the health post-health center (hospital) referral linkage to increase institutional delivery and ANC coverage.

Promote maternal education through adult education and Increase IYCF message through available local Medias.

\section{Abbreviations}

ANC: Antenatal Care; BF: Breast Feeding; BMI: Body Mass Index; CF: Complimentary Feeding; DHS: Demographic Health Survey; EBF: Exclusive Breast Feeding; EDHS: Ethiopian Demography Health survey; EPI: Expand Program of Immunization; IYCF: Infant and Young Child Feeding; IYCFP: Infant and Young child Feeding Practice; OR: Odds Ratio; PI: Principal Investigator; PNC: Post natal Care: UNICEF: United Nation Children's Fund; WHO: World Health Organization; TICF: Time of initiating complementary feeding.

\section{Authors' Contribution}

EE has conceived the study, carried out the overall design and execution of the study, performed data collection and statistical analysis. MK, EW OS and SR has critically revised the design of the study, data collection techniques and helped the statistical analysis. MK has drafted the manuscript. All 
authors read and finally approved this manuscript for submission.

\section{Competing Interests}

This is to declare that we all authors have no competing interest in this research title.

\section{Availability of Data and Materials}

The datasets supporting the conclusions of the study are included in the article. Any additional data will be available on request. The datasets used and/or analyzed during the current study are available from the corresponding author on reasonable request.

\section{Ethics Approval and Consent to Participate}

The study was approved by the Institutional Review Board of Collage of Medical and Health Sciences, Samara University. A letter of support was obtained from Assayita district health office. All results of this research were based on the use of primary data and the data collection was performed prospectively. Therefore, informed written consent form from the study participants was obtained and the study was conducted in accordance with the ethical standards of the institutional and National research committee. The study also adhered to the declarations of Helsinki.

\section{Funding}

The study was funded by Samara University.

\section{Acknowledgements}

We would like to thank all respondents for giving us valuable information. Our special thanks and appreciation also goes to Samara University department of public health for funding support. We also extend our gratitude to Afar Regional Health Bureau and Assayita Woreda Health office for their support throughout the work. Finally, we would like to thank all data collectors and supervisors who have given their precious time to collect the necessary data.

\section{References}

[1] Lamberti. L and Black. R, 2011. Breast-feeding and risk for diarrhea, morbidity, and mortality. BMC Public Health, P. 11: 15.

[2] Ali D. Alive \& Thrive, 2010. Practices, IYCF practices, beliefs, and influence in SNNP region Ethiopia, Survey report.

[3] EH NRI. Assessment of status of infant and young child feeding practice, policy and programmatic achievements and gap. Workshops on Assessment of status of infant and young child feeding practice, policy and programmatic achievements and gaps, November 12-16, 2012, Addis Ababa Ethiopia.

[4] Victora C, Hallal P, Blossner M and Shrimpton R. Worldwide timing of growth faltering: revisiting implications for interventions, Journal of Pediatrics. 2010; 125: 473-480.

[5] Dewel G. Systematic review of the efficacy and effectiveness of complementary feeding interventions in developing countries, Maternal and Child Nutrition, 2008; 4: 24-85.

[6] WHO/UNICEF (2006) Infant and Young Child Feeding Counseling: An Integrated Course. Participant's Manual.

[7] WHO/UNICEF/USAID/FANTA/IFPRI (2008) Indicators for Assessing Infant and Young Child Feeding Practices: Part 1: Definitions. World Health Organization, Geneva.

[8] WHO/UNICEF/USAID/FANTA/IFPRI (2010) Indicators for Assessing Infant and Young Child Feeding Practices: Part 2: Measurement. World Health Organization, Geneva.

[9] UNICEF. Nutrition: Breastfeeding and complementary feeding. 2015.

[10] PAHO/WHO (2002) Guiding Principles for Complementary Feeding of the Breastfed Child. Pan American Health Organization/World Health Organization, Washington DC.

[11] Kimani-Murage, E., et al. (2011) Patterns and Determinants of Breastfeeding and Complementary Feeding Practices in Urban Informal Settlements, Nairobi Kenya. BMC Public Health, 11, 396.

[12] Jones, G. (2003) How Many Child Deaths Can We Prevent This Year? The Lancet, 362, 65-71.

[13] Black RE, Victora CG, Walker SP, et al. Maternal and child under nutrition and overweight in low-income and middleincome countries. Lancet 2013; 382: 427-51.

[14] Edmond KM, Zandoh C, Quigley MA, et al. Delayed breastfeeding initiation increases risk of neonatal mortality. Pediatrics 2006; 117: e38 0-e386.

[15] Setegn T, Gerbaba M, Belachew T. Determinants of timely initiation of breastfeeding among mothers in Goba Woreda, South East Ethiopia: a cross sectional study. BMC Public Health 2011; 11: 1 .

[16] Baker EJ, Sanei LC, Franklin N. Early initiation of and exclusive breastfeeding in large-scale community-based programmes in Bolivia and Madagascar, J Health Popul Nutr 2006; 24: 530-9.

[17] Ogbo FA, Page A, Idoko J, et al. Diarrhoea and suboptimal feeding practices in Nigeria: evidence from the National Household surveys. Paediatr Perinat Epidemiol 2016; 30: 346-55.

[18] Elizabeth KE. Feeding of young infants and children in exceptionally diffcult circumstances (like, HIV and humanitarian emergencies). Solution exchange $\mathrm{MCH}$ Community Newsletter Breastfeeding Month Special. 2008.

[19] World Health Organization. Exclusive breastfeeding for six months best for babies everywhere, 2011, 2012.

[20] World Health Organization. Infant and young child feeding, 2017.

[21] Begum K, Dewey KG. Impact of early initiation of exclusive breastfeeding on new born deaths, 2010. 
[22] Central Statistical Agency (CSA) [Ethiopia] and ICF. 2016. Ethiopia Demographic and Health Survey 2016: Key Indicators Report. Addis Ababa, Ethiopia, and Rockville, Maryland, USA. CSA and ICF.

[23] Ethiopia Federal Ministry of Health, Ethiopian national strategy form infant and young child feeding. 2004.

[24] Miller NP, Amouzou A, Tafesse M, et al. Integrated community case management of childhood illness in Ethiopia: implementation strength and quality of care. Am J Trop Med Hyg 2014; 91: 424-34.

[25] Labbok MH. Global baby-friendly hospital initiative monitoring data: update and discussion. Breastfeed Med 2012; 7: $210-22$.

[26] Sharma IK, Byrne A. Early initiation of breastfeeding: a systematic literature review of factors and barriers in South Asia. Int Breastfeed J 2016; 11: 1.

[27] Horta BL, Victora CG. Long-term effects of breastfeeding: a systematic review. 2013.

[28] Horta BL, Loret de Mola C, Victora CG. Breastfeeding and intelligence: a systematic review and meta-analysis. Acta Paediatrn 2015; 10.

[29] Kramer MS, Kakuma R. The optimal duration of exclusive breastfeeding. Protecting infants through human milk, Springer, 2004: 63-77 4: 14-19.

[30] Dewey KG, Adu-Afarwuah S. Systematic review of the effcacy and effectiveness of complementary feeding interventions in developing countries. Matern Child Nutr 2008; 4 (Suppl 1): 24-85.

[31] Kume Abera. Infant and young child feeding practices among mothers living in Harar Ethiopia Harare bulletin of health science.

[32] F. Yonas et al. Infant and Young Child Feeding Practice Status and Associated Factors among Mothers of under 24-MonthOld Children in Shashemene Woreda, Oromia Region, Ethiopia July 2015 Volume 2|e1635.

[33] Birhane Yitages. Timely initiation of complementary feeding and associated factors children aged 6 to 12 months in northern Ethiopia. An institution based cross sectional study. BMC public health 2013, 13: 1050.

[34] Tamiru, D., Bogale, B. and Merdekios, B. (2013) Breastfeeding Patterns and Factors Associated with Exposure to SubOptimal Breastfeeding Practices in Rural Communities of Arba Minch Zuria, Ethiopia. Global Health Perspectives, 1, $105-112$.

[35] Yeshalem Mulugeta Demilew, Tadese Ejigu Tafere and Dereje Berhanu Abitew, Infant and young child feeding practice among mothers with 0-24 months old children in Slum areas of Bahir Dar City, Ethiopia, International Breastfeeding Journal (2017) 12: 26.

[36] Sasie SD, Oljira L and Demena M. Infant and Young Child Feeding Practice and Associated Factors among Mothers/Caretakers of Children Aged 0-23 Months in Asella Town, South East Ethiopia. J Fam Med. 2017; 4 (5): 1122.

[37] Wamani, H., Peterson, S., Tylleskar, T. and Tumwine, J. K. (2005) Infant and Young Child Feeding in Western Uganda: Knowledge, Practices and Socio-Economic Correlates. Journal of Tropical Pediatrics, 51, 356-361. http://dx.doi.org/10.1093/tropej/fmi048.

[38] Victor, R. (2012) Infant and Young Child Feeding Practices among Children Aged 0-23 Months in Tanzania. Maternal and Child Nutrition Journal.

[39] Kabir I, Khanam M, Agho KE, et al. Determinants of inappropriate complementary feeding practices in infant and young children in Bangladesh: secondary data analysis of Demographic Health Survey 2007. Matern Child Nutr 2012; 8 (Suppl 1): 11-27.

[40] Demewoz Haile, Muluken Azage, Tegegn Mola and Rochelle Rainey: Exploring spatial variations and factors associated with childhood stunting in Ethiopia: spatial and multilevel analysis, BMC Pediatrics (2016) 16: 49.

[41] Disha A, Tharaney M, Abebe Y, Alayon S, and Winnard K. Factors associated with infant and young child feeding practices in Amhara Region and nationally in Ethiopia: Analysis of the 2005 and 2011 Demographic and Health Surveys. Washington, DC: Alive \& Thrive; 2015. 